\title{
Antimicrobials for Preterm Birth Prevention: An Overview
}

\author{
Akila Subramaniam, ${ }^{1,2}$ Adi Abramovici, ${ }^{1}$ William W. Andrews, ${ }^{1}$ and Alan T. Tita ${ }^{1}$ \\ ${ }^{1}$ Department of Obstetrics and Gynecology, Division of Maternal-Fetal Medicine, University of Alabama at Birmingham, \\ 1700 6th Avenue South, Birmingham, AL 35223, USA \\ ${ }^{2}$ Department of Obstetrics and Gynecology, University of Alabama at Birmingham, Women and Infants Center, \\ 1700 6th Avenue South, Birmingham, AL 35223, USA
}

Correspondence should be addressed to Akila Subramaniam, akila.subramaniam@gmail.com

Received 2 June 2011; Accepted 21 November 2011

Academic Editor: Joseph Hwang

Copyright (C) 2012 Akila Subramaniam et al. This is an open access article distributed under the Creative Commons Attribution License, which permits unrestricted use, distribution, and reproduction in any medium, provided the original work is properly cited.

\begin{abstract}
Objective. Preterm birth (PTB) remains a major cause of neonatal morbidity and mortality. The association between PTB and infection is clear. The purpose of this report is to present a focused review of information on the use of antibiotics to prevent PTB. Methods. We performed a search of the PubMed database restricted to clinical trials or meta-analyses published in English from 1990 through May 2011 using keywords “antibiotics or antimicrobials" and "preterm." Results. The search yielded 67 abstracts for review. We selected 31 clinical trials $(n=26)$ or meta-analysis $(n=5)$ for further full-text review. Discussion of each eligible clinical trial, its specific inclusion criteria, antibiotic regimen used, and study results are presented. Overall, trials evaluating antibiotic treatment to prevent preterm birth have yielded mixed results regarding any benefit. Conclusion. Routine antibiotic prophylaxis is not recommended for prevention of preterm birth.
\end{abstract}

\section{Introduction}

Preterm birth (PTB) continues to be a leading cause of neonatal morbidity and mortality. Defined as birth prior to 37 weeks of gestation, PTB accounts for over $35 \%$ of health care spending for infants and $10 \%$ of health care spending for children in the United States [1]. The incidence of PTB has been estimated to be $12-13 \%$ in the United Statesor approximately 467,000 annual live births-significantly higher compared to European and other developed countries with incidence at 5-9\% [2-4]. In fact, from 1996 to 2006, there was a steady increase in PTBs in the US to a peak at $12.8 \%$-with the first decline in 2007 with a PTB rate of $12.7 \%[5,6]$. Moreover, despite advances in management of preterm labor and care of preterm infants, PTB still constitutes $75 \%$ of neonatal mortality and $50 \%$ of long-term neurologic impairment in children [7]. Spontaneous PTB (SPTB) accounts for the large majority (65-85\%) of PTB and is strongly associated with infection [8]. As a result, considerable effort has been dedicated to evaluate antimicrobial therapy as an intervention to prevent PTB. The purpose of this report is to review the role of infection in the epidemiology of PTB specifically focusing on the use antibiotics to prevent PTB.

\section{Methods}

We implemented a descriptive and analytic literature review of antimicrobial therapy to prevent PTB; our objective was not to conduct a synthetic meta-analysis (with summary estimates). Rather, it was to identify relevant contemporary articles on infection and antimicrobial therapy for PTB prevention. We searched the PubMed database restricted to clinical trials or meta-analyses published in English since 1990. Keywords for this search included "antibiotics or antimicrobials" and "preterm" yielding 67 abstracts for review by 2 authors. Of these 67 abstracts, only 31 eligible clinical trials $(n=26)$ or meta-analysis $(n=5)$ evaluated the use of antimicrobials to prevent PTB, and this was retrieved for further full-text review. For each eligible study we present its specific inclusion criteria, the antibiotic regimen used, and the study results. 


\section{Background}

3.1. Epidemiologic Overview, Subtypes, and Mechanisms of Preterm Birth. Part of the difficulty in preventing PTB is its multifactorial etiology. The relative frequencies of the 3 major subcategories of preterm birth are the following: spontaneous preterm labor (50\%), delivery for maternal or fetal indications (30\%), and preterm premature rupture of membranes or PPROM $(20 \%)[8,9]$. Compared with other ethnicities, black women are at disproportionately increased risk for PTB; risks are generally lower among Hispanic women [8]. Black women have double the rates of PTB as white women and women with a previous preterm delivery have a 15-50\% recurrence risk [8]. Increasing prevalence of assisted reproductive technology and multiple gestations [10], as well as maternal and fetal indications including hypertension, diabetes, and intrauterine growth restriction contribute to rates of PTB [11].

Late preterm births (defined as birth between 34 and 36 weeks) contribute over $70 \%$ of PTB [6]. While these births carry less risk of neonatal morbidity and mortality than earlier births, they still carry a greater risk of neonatal impairment compared to term births.

Overall, key risk factors for preterm birth include age, race, nutritional status, a history of a previous PTB, multiple gestation, smoking, altered vaginal bacterial flora, and presence of infection [12]. Obesity itself is associated with hypertension and preeclampsia leading to indicated preterm birth but has been noted to be protective of spontaneous preterm birth [12].

How these risk factors contribute to the pathogenesis of spontaneous preterm labor and preterm premature rupture of membranes (PPROM) is largely unknown. Proposed mechanisms of term labor include progesterone withdrawal, oxytocin initiation, decidual activation, and activation of the fetal immune response [8]. The first two pathways have not been clearly elicited in humans, but inflammation-mediated decidual activation seems plausible. In this theory, infection, inflammation, or hemorrhage stimulates the innate immune system; chemokines and cytokines induce prostaglandins and other matrix degrading enzymes causing uterine contractility and degradation of extracellular matrix leading to labor and rupture of membranes $[12,13]$. Activation of the fetal immune response could have a similar impact [12]. While this could similarly be implicated in preterm labor and PROM, other mechanisms include infection, inflammation, uteroplacental ischemia of hemorrhage, uterine overdistension, or psychosocial stress [12].

3.2. Infection and Preterm Birth. Intrauterine infection is estimated based on studies of amniotic fluid and cultures to be associated with approximately $25-40 \%$ of PTB-the association stronger, the earlier the gestation at birth [12]. The different types of infection involving the maternal fetal interface include deciduitis, chorioamnionitis, villitis, and funisitis [12]. Although infection is noted to be extremely common in preterm delivery, it is often chronic, subclinical, and asymptomatic (apart from labor). In one study of 602 women delivered by cesarean section prior to membrane rupture,
$121(20.1 \%)$ had positive membrane culture; $50 \%$ of these women also had positive amniotic fluid culture-often with the same organism [12]. In another study of the placentas of 446 mother-infant pairs, women with a SPTB were significantly more likely to have acute inflammation of the membranes, chorionic plate, and the umbilical cord than women with indicated preterm births [13].

The pathogenesis of SPTB in the setting of subclinical infection is not fully elucidated-the current understanding is presented in Figure 1 [12]. Bacterial invasion of maternal and fetal tissues causes release of endo and exotoxins. These substances activate both a maternal response stimulating the release of cytokines such as tumor necrosis factor alpha (TNF-alpha), interleukin-1alpha, interleukin-1 $\beta$, interleukin-6, interleukin-8, granulocyte colony-stimulating factor (G-CSF), and other factors [12, 14-18]. These active substances stimulate the production and release of prostaglandinscausing uterine contractility - and neutrophil activity and thereby synthesis and release of metalloproteinases-causing rupture of membranes and remodeling of collagen in the cervix [19-21]. A fetal response also may occur, in which infection stimulates the production of corticotropin-releasing hormone (from the fetal hypothalamus and placenta) causing an increase in fetal corticotropin and thereby fetal cortisol also stimulating prostaglandins [22].

Multiple inflammatory markers have been noted to be elevated in amniotic fluid, the serum, or vaginal and cervical secretions. These include amniotic fluid interleukin- 6 concentration interleukin-1, interleukin-8, G-CSF, and TNFalpha, ferritin, and fetal fibronectin; however, none of these markers have been found useful in predicting preterm delivery in routine clinical practice $[12,23]$.

Given the strong association between SPTB and infection, prophylactic antibiotics seem a logical preventative strategy for PTB. This review focuses on the specific infections associated with PTB and the effectiveness and safety of antibiotic prophylaxis.

3.3. Organisms and Mechanisms of Infection. In order to evaluate the effectiveness of specific antibiotics used to prevent $\mathrm{PTB}$, it is important to consider the microbes implicated in associated intrauterine infections. Organisms most commonly gain access to the uterus and pregnancy by ascending from the vagina and cervix [12]. Less frequent pathways include iatrogenic inoculation during amniocentesis or chorionic villus sampling, hematogeneous spread through uterine and placental blood flow or through descending infection from the abdominal cavity and fallopian tubes to the chorioamnion. Ascending infection extends to the choriodecidual space and may ultimately invade the membranes, amniotic fluid, and fetus [12]. The exact timing of infection in relation to pregnancy is not fully elucidated but has been hypothesized to occur early in gestation or even in the preconceptional period [24].

The most commonly isolated organisms include microbes with low virulence: Ureaplasma urealyticum, Mycoplasma hominis, Gardnerella vaginalis, peptostreptococcus, and bacteroides species - all organisms of vaginal flora [2528]. Ureaplasma and Mycoplasma species are the most 


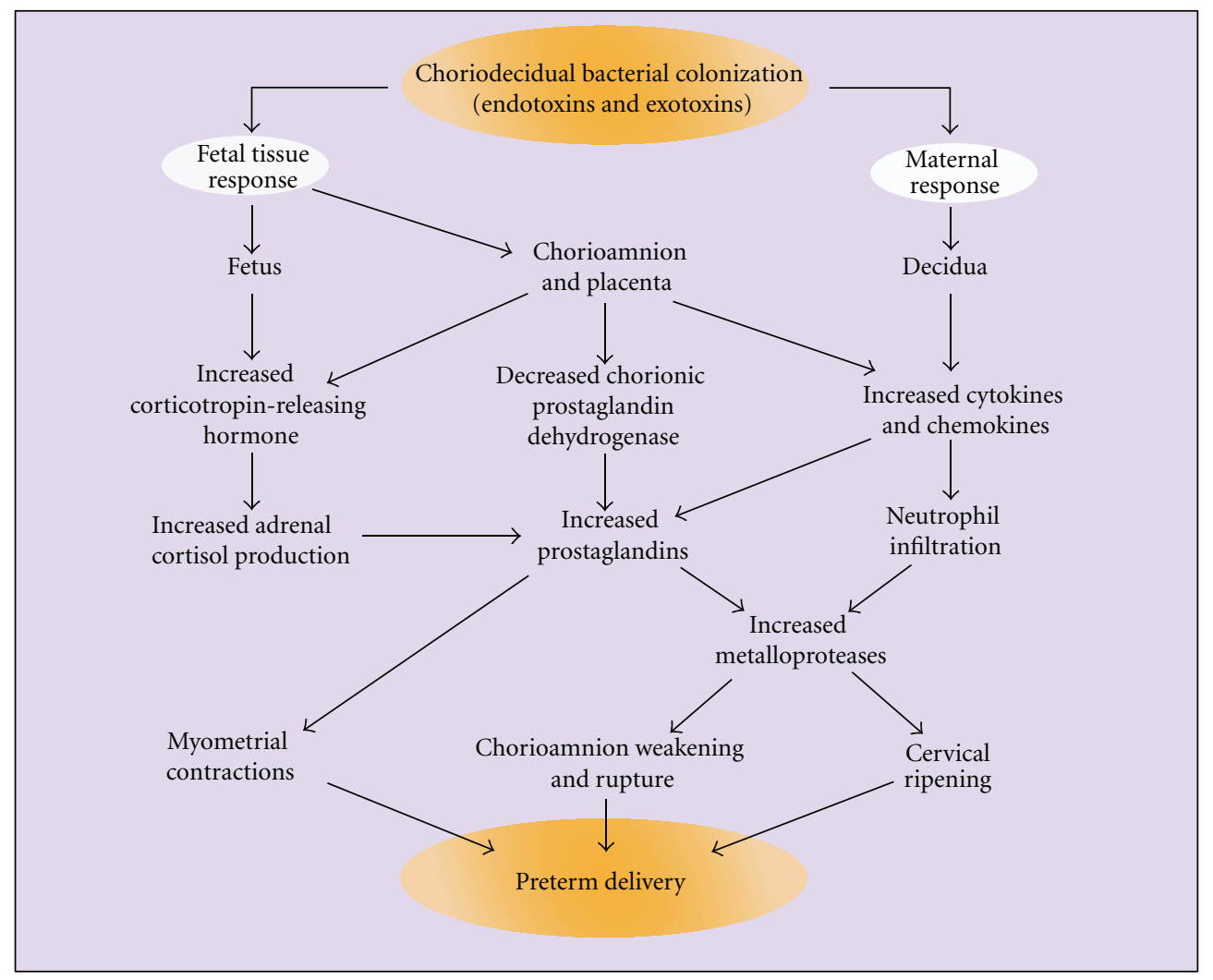

FIgURe 1: Potential Pathways from choriodecidual bacterial colonization to preterm delivery [12].

common microbes cultured from placental tissue and fluids obtained from patients with histologic and clinical chorioamnionitis. In a study of umbilical cord blood in preterm 23-32-week births, Goldenberg et al. showed that Ureaplasma urealyticum and/or Mycoplasma hominis were present in $23 \%$ of cultures - especially in women undergoing spontaneous compared to indicated preterm delivery (34.7\% versus $3.2 \%, P<0.001)[29]$.

Bacterial vaginosis (BV) is also commonly associated with PTB. Due to an interruption in normal vaginal flora, it is characterized by a reduction in lactobacillus and an overgrowth of other anaerobic bacteria such as gardenerella, bacteroides, and mobiluncus. Its diagnosis is based on either Amsel's criteria (taking into account vaginal $\mathrm{pH}$, presence of discharge, amine odor, or presence of clue cells) or Nugent's score (a gram stain scoring system). Bacterial vaginosis is present in 15-42\% of pregnant women and confers a 2 to 4 fold independent increase in the risk of spontaneous preterm birth and PROM [30-33]. In a meta-analysis including 18 studies, Leitich et al. shows that the associated risk is greater when BV is diagnosed at earlier gestation [33]. The alterations in vaginal flora by bacterial vaginosis may also increase susceptibility to other ascending genital tract infections.

Sexually transmitted infections including Trichomonas vaginalis, Neisseria gonorrhoeae, Chlamydia trachomatis and syphilis have also been associated with preterm delivery and intrauterine infection [34-36]. Numerous small studies have shown adverse outcomes associated with trichomoniasis including PTB, PROM, and low birth weight [34]. In the largest study of trichomoniasis during pregnancy, Cotch et al. studied over 12,000 women and noted a modest 1.3 odds ratio of preterm delivery (95\% CI 1.1-1.4) [34]. Other genitourinary microbes such as Group B streptococcus and Escherichia coli have also been described in intrauterine infection and consequent preterm delivery [12].

Nongynecologic infections have also been studied in PTB including pneumonia, pyelonephritis, periodontitis, and gastrointestinal infections $[37,38]$. The mechanism of action of these infections is thought to be an acute inflammatory response, but for periodontal disease it is hypothesized to be transient bacteremia and hematogenous spread to the placenta, chorioamnion, and fetus [8]. Viral infections such as varicella and influenza have been associated with PTB; however, the role of viral infection in PTB but has been inadequately studied $[8,39,40]$.

\section{Results and Comments}

4.1. Antibiotic Therapy to Prevent PTB. Given the abounding data associating various infections with PTB, it is natural to consider the use of antibiotic and other antimicrobial therapy to prevent PTB. Studies have investigated the use of different antimicrobials both as empiric therapy providing broad coverage to prevent or treat infection and as targeted therapy against specific infections. In addition, the timing of 
antibiotic therapy in relation to pregnancy varies. Characteristics and findings from individual studies are presented below and summarized in Tables 1 and 2 .

4.1.1. Empiric Antibiotic Therapy. Macrolides (commonly erythromycin, azithromycin), beta-lactams, clindamycin, and metronidazole have been used in isolation or in combination as empiric therapy to prevent PTB. Macrolides and other bacteriostatic agents have been advocated since they suppress bacterial virulence as opposed to bactericidal agents such as penicillins, which theoretically may worsen outcomes for preterm infants by releasing bacterial endotoxins [60]. Combination antimicrobials provide empiric coverage against the common microbes including ureaplasmas, gramnegative rods, and anaerobes.

Beta-Lactams. Three randomized controlled studies have focused solely on the penicillin family of antimicrobials and PTB. Newton et al. failed to show any benefit in terms of days gained, neonatal outcome, or gestational age in a double-blind trial of the combination of ampicillin/sulbactam with indomethacin-a nonsteroidal anti-inflammatory tocolytic - in the setting of magnesium tocolysis [41]. Similarly, Cox et al. randomized 78 women in preterm labor with intact membranes at 24 to 34 weeks of gestation to either parenteral ampicillin ( 2 grams $)+$ sulbactam ( 1 grams) for 2 days followed by oral amoxicillin-clavulanic acid $(250 \mathrm{mg})$ for 5 days $(n=39)$, or to similar placebo $(n=39)$. Steroids or tocolysis were not administered. There was no difference in the mean gestational age at delivery $(34.2 \pm 0.7$ versus $34.1 \pm$ 0.9 , not significant); other perinatal outcomes were similar [42]. Finally, Gordon described similar findings among 117 women in preterm labor randomized to receive 2 grams of parenteral ceftizoxime or placebo for 5 days [43]. The primary outcome of interest, mean interval to delivery, was not significantly different between the two groups $(34.5 \pm 21.1$ days versus $34.6 \pm 24.5$ days, $P=0.99$ ). In these three studies, regardless of the use of tocolysis or steroid administration, no significant benefit was associated with the use of penicillins.

Clindamycin. While studies of beta-lactams have not been promising, a small-randomized trial by McGregor et al. showed some benefit of clindamycin. Women on tocolysis for preterm labor at gestational age less than 34 weeks were randomized to receive either placebo versus parenteral followed by oral clindamycin for 7 days. Patients treated with clindamycin at gestational ages less than 32 weeks were more likely to have increased duration of pregnancies (35 versus 25 days, $P=0.02$ ). Moreover, in a subgroup analysis, those women diagnosed with bacterial vaginosis and then treated with clindamycin were more likely to have longer pregnancy latency, increased birth weights, and increased gestational age at delivery [44]. Lamont et al. described up to a $60 \%$ reduction of $\mathrm{PTB}$ with the use of $2 \%$ clindamycin cream in patients with abnormal genetic flora (4\% versus 10\%; $P<$ $0.03)$ [45]. However, other studies of vaginal clindamycin specifically for bacterial vaginosis-discussed below-have been contradictory [46]. Some studies have even suggested increased risk of bacterial resistance and neonatal morbidity $[61,62]$. Given the disparity of findings, the role of clindamycin in PTB prevention remains inconclusive.

Azithromycin. Few studies have been performed solely on macrolides. In the large 2200 patient APPLe study conducted in Southern Malawi, van den Broek et al. conducted a placebocontrolled trial of oral azithromycin (1 gram) given at 1624 and again at 28-32-weeks, gestation to unselected women. Findings showed no significant differences in outcomes between the two groups regarding PTB (16.8\% versus $17.4 \%$, odds ratio $0.96(0.76-1.21))$ or other perinatal outcomes including perinatal mortality. Further inclusion of this study in a meta-analysis with seven other studies also failed to show any salutary effect on PTB [47].

Combination Antibiotic Regimens. It seems that, regardless of the antibiotic of choice, gestational age at administration, route of administration, days of therapy, or repeat dosages of antibiotics, no single agent has been noted to be efficacious in the prevention of PTB. Thus, trials involving empiric combination antibiotic regimens are more prevalent in the obstetric literature.

Beta-lactams Plus Metronidazole. Norman et al. randomized 81 women in preterm labor to receive parenteral ampicillin or oral amoxicillin and metronidazole for 5 days $(n=43)$ versus no antibiotic treatment $(n=38)$. All women were treated with indomethacin and hexoprenaline for tocolysis and betamethasone for fetal lung maturity. Women receiving antibiotics had significantly prolonged pregnancies (median 15 versus 2.5 days, $P=0.04$ ). In addition, patients in the control group had significantly increased risks of necrotizing enterocolitis (5 versus $0, P=0.02$ ) [48].

Similar benefit of ampicillin plus metronidazole was noted by Svare et al. in a randomized study of 112 women in preterm labor with intact membranes between 26- and 34 -weeks gestation. Women receiving parenteral ampicillin for 24-hour followed by penicillin agent pivampicin 500 milligrams orally for 7 days) and metronidazole for 7 days were compared to controls receiving placebo. Those receiving antibiotics had prolonged pregnancy (47.5 days versus 27 days, $P<0.05)$, higher mean gestational age at delivery ( 37 versus 34 weeks, $P<0.05)$, decreased incidence of preterm birth $<37$ weeks ( $42 \%$ versus $65 \%, P<0.05$ ), and lower rates of admission to neonatal intensive care unit (40\% versus $63 \%, P<0.05$ ) [49]. Although both studies noted some benefit with the beta-lactam plus metronidazole antibiotic regimens, no larger studies have been performed. Moreover, improved outcomes could have been secondary to tocolysis and/or antenatal steroid therapy.

Beta-Lactams and Macrolides. Trials of beta-lactams combined with macrolides have yielded mixed results. In a blinded study by Newton et al., 103 women between 24-35 weeks' gestation in preterm labor undergoing tocolysis were randomized to receive either parenteral ampicillin and oral erythromycin for 7 days or placebo. No statistical differences 


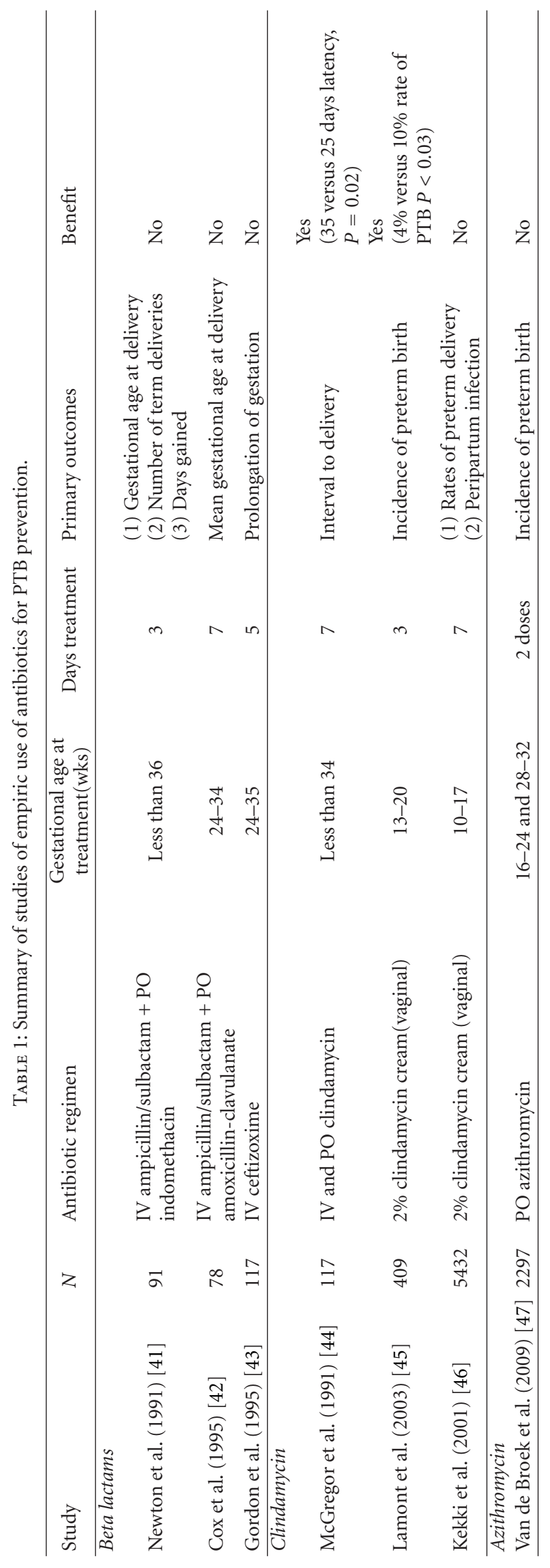




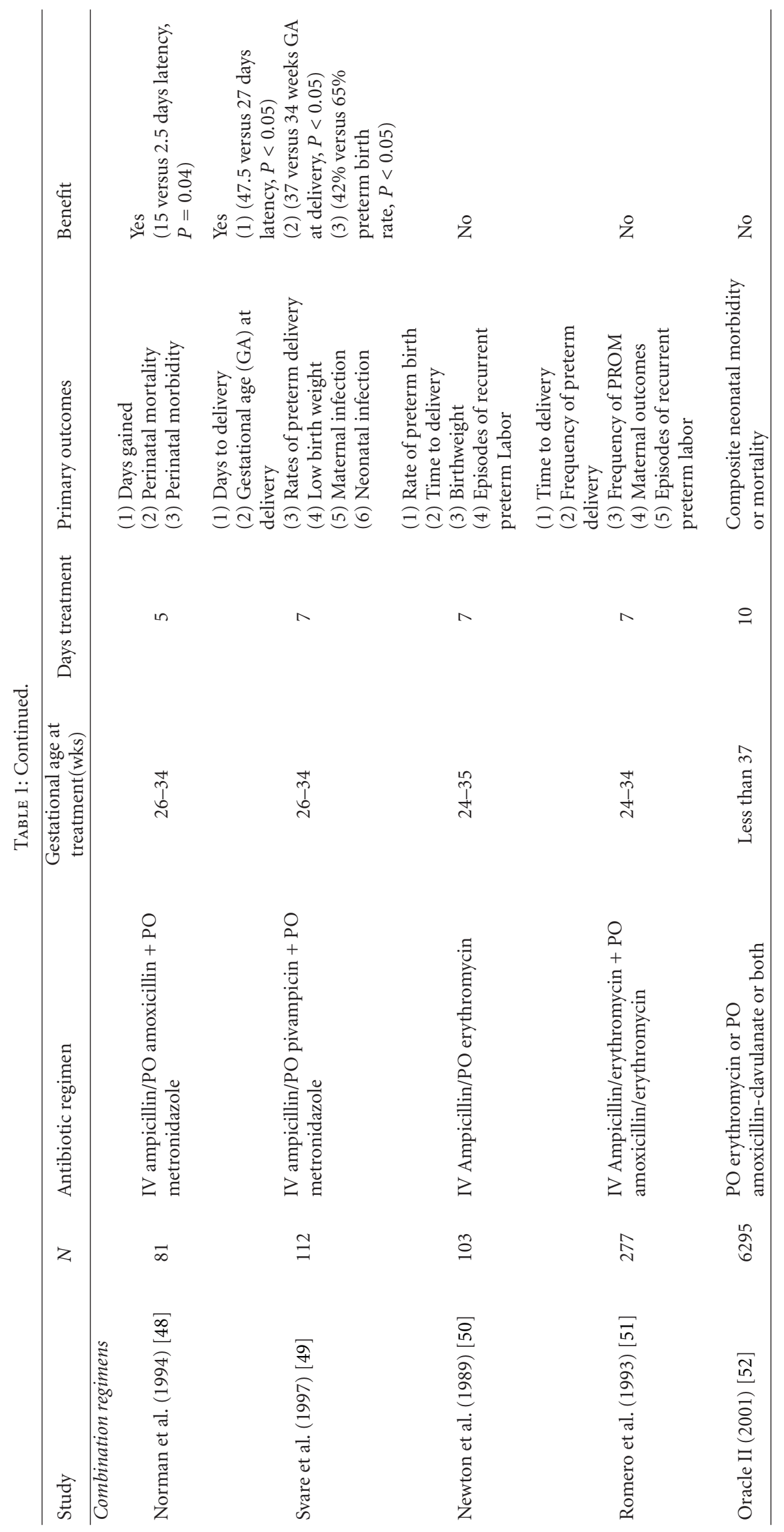




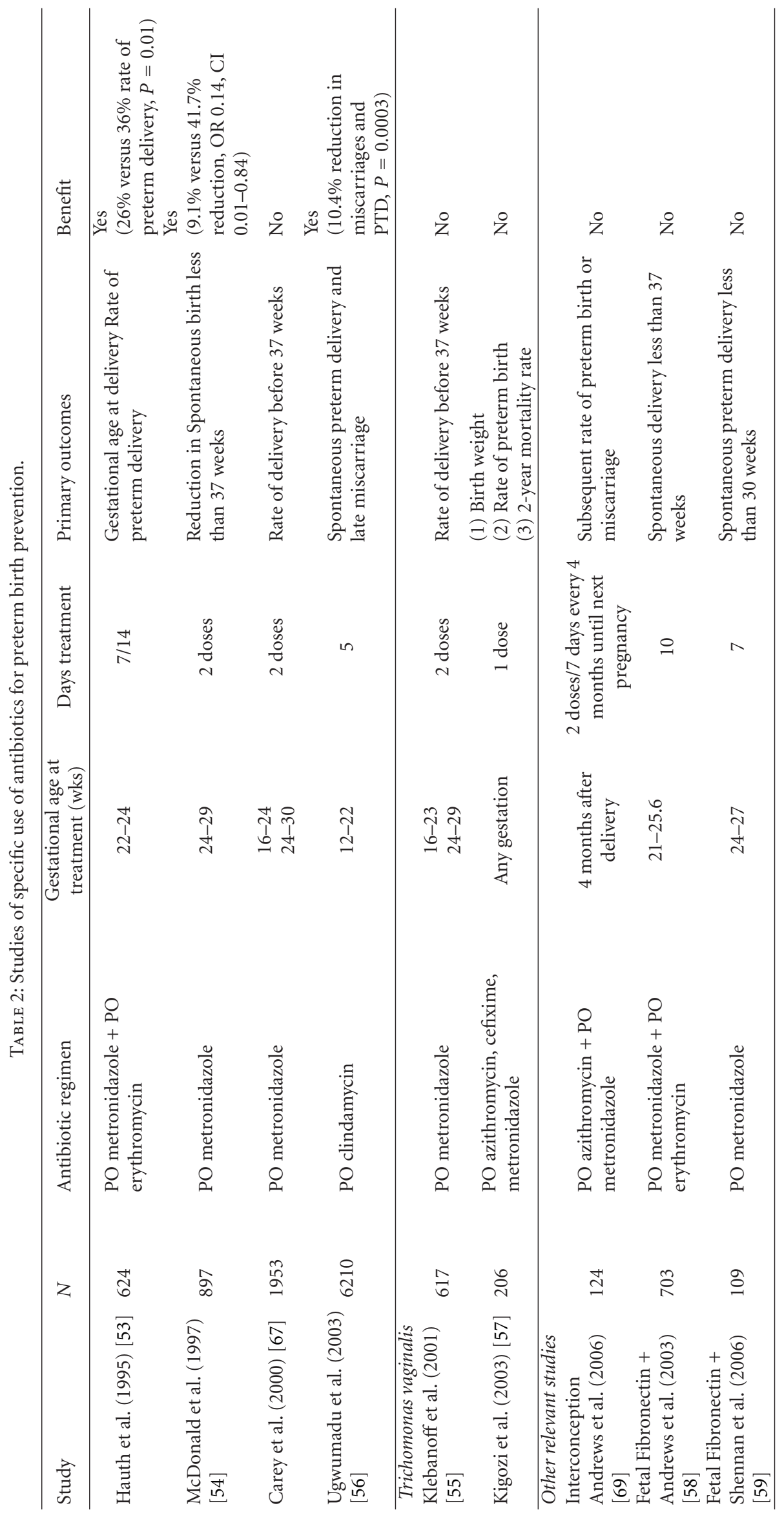


were noted between groups in terms of time to delivery, frequency of preterm birth, or birth weight [50].

Similarly no differences were noted by Romero et al. in a multicenter, randomized trial from the Maternal Fetal Medicine Units Network [51]. In this study of 277 women at six centers, 133 received intravenous ampicillin and erythromycin for 48 hours followed by oral amoxicillin and erythromycin for 5 days; the control group received placebo. No statistically significant differences were noted in time to delivery, frequency of preterm delivery $<37$ weeks, frequency of premature rupture of membranes, or neonatal outcomes including Neonatal Intensive Care Unit (NICU) admission, respiratory distress, and sepsis. Further trials by Watts et al. and Oyarzún et al. have also failed to demonstrate benefit to penicillins combined with macrolides [63, 64]. Furthermore, in the Oracle II trial with over 6000 women in preterm labor, erythromycin, amoxicillin-clavulanic acid as compared with placebo was not associated with any maternal or fetal benefit [52]. Results of the longterm followup on women in the Oracle II trial suggest an increase in cerebral palsyindicating that routine beta-lactam and macrolide antibiotics solely for PTB prevention may be ill-advised.

Macrolides and Metronidazole. One large-scale study has directly evaluated metronidazole with macrolides. Hauth et al. in a double blind, randomized controlled study evaluated the efficacy of treating patients with bacterial vaginosis in the 2nd trimester of pregnancy (22-24 weeks). 624 women with a history of a prior spontaneous preterm birth were allocated in a 2:1 ratio in an antibiotic group $(n=433)$ or placebo (191). The antibiotic regimen consisted of metronidazole 250 milligrams three times a day for 7 days and erythromycin 333 milligrams three times a day for 14 days. Patient receiving antibiotics had a lower rate of preterm delivery ( $26 \%$ versus $36 \%, P=0.01)$. Among the women with bacterial vaginosis included in the Hauth trial, antibiotics significantly reduced the incidence of PTB ( $31 \%$ versus $49 \%, P=0.006$ ) leading to the conclusion that antibiotic therapy may reduce preterm delivery in patients at risk for premature delivery with bacterial vaginosis [53]. These patients, however, were treated earlier in pregnancy than any of the earlier discussed trials and were all known to have documented infection with bacterial vaginosis.

Meta-Analyses. In a Cochrane Review, King et al. included 11 studies (the largest of which was the Oracle II trial) and a total of 7428 women. There was a reduction in maternal infection (relative risk 0.74, 95\% CI 0.64-0.87), but no statistically significant differences in mean gestational age at delivery, frequency of preterm birth, and neonatal outcomes including mortality. In addition, no differences were noted in a subgroup analysis between the types of antibiotics [65]. Furthermore, no subgroup analysis was presented for patients receiving metronidazole in the first half of pregnancy.

Similarly, a more recent meta-analysis performed by Simcox et al. in 2007 identified 17 randomized controlled trials comparing antibiotics to placebo in asymptomatic nonlaboring women with a risk of PTB (previous PTB, positive fetal fibronectin, or abnormal vaginal flora). No significant association was noted in the reduction of PTB, the antimicrobial administered, or gestational age at treatment [66]. In summary, while small-scale studies have shown some benefit to antibiotic prophylaxis-especially in the case of beta-lactams and metronidazole - no advantages, efficacy, or prevention of PTB has been consistently presented to support routine antibiotic therapy. The question then arises if there is any efficacy to treating specific infections with targeted therapy.

\subsubsection{Specific Antibiotic Therapy}

Bacterial Vaginosis. Several studies have specifically evaluated the role of antibiotics prophylaxis for preterm delivery in the setting of bacterial vaginosis. Hauth et al. in a double blind, randomized controlled study described above concluded that antibiotic therapy may reduce preterm delivery in patients at risk for premature delivery with bacterial vaginosis [53].

McDonald et al. revisited the possibility of a reduction in the risk of SPTB with metronidazole treatment of women with heavy growth of Gardnerella during mid-pregnancy. Patients identified with heavy growth of Gardnerella or a gram stain indicative of bacterial vaginosis were randomized to receives oral metronidazole 400 milligrams or placebo twice daily for two days at 24-week gestation and then again at 29 weeks [54]. No differences were noted in overall PTB or SPTB; however, in a subgroup analysis of women with a previous PTB, a significant reduction in SPTB (9.1\% versus $41.7 \%$, OR 0.14 CI 0.01-0.84) was noted [54].

Given these positive results, a larger Maternal Fetal Medicine Units Network trial $(n=1953)$ was performed using metronidazole to prevent PTB by treating asymptomatic bacterial vaginosis between 16 and 24 weeks of gestation [67]. Asymptomatic bacterial vaginosis (absence of itching, odor or discharge) was diagnosed by Nugent's criteria and treated with two doses of metronidazole (2 grams) or placebo. There were no differences in the rates of preterm delivery $(12.2 \%$ versus $12.5 \%$, relative risk $1.0,95 \%$ CI $0.8-1.2)$, spontaneous rupture of membranes $(4.2 \%$ versus $3.7 \%$ ), delivery before 32 weeks ( $2.3 \%$ versus $2.7 \%$ ), or neonatal outcomes [67]. A meta-analysis of 15 trials with over 5800 women with bacterial vaginosis that examined the effect of metronidazole to treat bacterial vaginosis on preterm birth $<37$ weeks showed no risk reduction (odds ratio $0.91,95 \%$ CI: 0.78-1.06) [68]. In this meta-analysis, however, there were no results presented regarding comparison between patients with symptomatic versus asymptomatic infection.

Trials have also yielded mixed results regarding intravaginal clindamycin for bacterial vaginosis. While Lamont showed some benefit regarding PTB in patients with abnormal vaginal flora, in a study of over 5000 women by Kekki et al., vaginal clindamycin did not decrease the rate of preterm deliveries (OR 1.3, 95\% CI 0.5,3.5) [45, 46]. Trials have similarly yielded mixed results on the role of oral clindamycin for antibiotic prophylaxis in the setting of bacterial vaginosis. Ugwumadu et al. studied patients at high risk for late miscarriage or SPTB-randomized women between 12 
and 22 weeks of gestation with bacterial vaginosis receiving oral clindamycin $300 \mathrm{mg}$ twice daily for five days or placebo. Women receiving clindamycin had significantly fewer late miscarriages or preterm deliveries (10.4\% percentage difference, $P<0.001$ ) [56]. No subgroup analysis of solely preterm birth was reported.

It is evident that there is no convincing data to support the routine use of metronidazole or clindamycin in the $2 \mathrm{nd}$ trimester of pregnancy in patients with bacterial vaginosis. While few small studies have shown some possible efficacy, larger trials have refuted any benefit. In fact, other trials have even noted deleterious effects of clindamycin on neonatal morbidity-especially noted to be infectious morbidity [61, $62]$.

Trichomonas Vaginalis. No benefit for PTB prevention has been shown for treatment of asymptomatic or symptomatic trichomoniasis. Klebanoff et al. randomly assigned 617 patients with asymptomatic trichomoniasis (defined as trichomonas on culture of vaginal secretions) to metronidazole (two doses of 2 grams 48 hours apart) versus placebo between 16 to 23 weeks of gestation and then again at 2429 -week gestation. Delivery prior to 37 weeks occurred in $19 \%$ of women treated with antibiotics and $10.7 \%$ of patients receiving placebo (relative risk 1.8, 95\% CI: 1.2-2.7, $P=$ 0.004) [55]. Thus, not only did they observe no benefit for treatment of asymptomatic trichomoniasis, intervention was found to be harmful.

Kigozi et al. similarly noted no benefit to treatment of trichomonas during pregnancy. In their randomized controlled trial women presumptively diagnosed with trichomonas were randomized to receive oral 1 gram azithromycin, 400 milligrams cefixime, and 2 grams metronidazole. Increased rates of low birth weight, preterm birth, and 2-year mortality were noted in patients treated for infection [57]. Again, intervention was noted to be harmful. However, it should be emphasized that these patients were treated at any gestational age at pregnancy and were not a high-risk population for PTB.

Timing of Antibiotic Administration. In addition to type and specificity of antibiotic regimens, the timing of antibiotic administration is an important consideration. Uterine infection may occur prior to conception, early in pregnancy or at later gestational age. Therefore, it has been postulated that the lack of benefit in some trials, may in part be due to late treatment when the mechanisms leading to PTB may already be established. Based on this hypothesis, Andrews et al. performed an interconceptional antibiotic randomized trial. Women with a SPTB prior to 34 weeks were randomized four months postpartum to receive oral azithromycin 1 gram twice (4 days apart) plus metronidazole 750 milligrams daily for 7 days $(n=59)$ or placebo $(n=65)$. The regimen was repeated every 4 months until next pregnancy. No statistical differences were observed between subsequent SPTB at less than 37, 35, or 32 weeks. Interestingly, patients in the antibiotic group were noted to have decreased mean birth weight and earlier mean delivery gestational age, albeit not statistically significant [69]. Therefore, additional welltailored trials are needed to further clarify this issue.

Antibiotics for Other Indications Associated with PTB. PTB may occur not only as a result of intrauterine subclinical infection, but also from systemic infection and PROM. In a Cochrane review of PROM, Kenyon analyzed 6800 subjects in 22 randomized controlled clinical trials. Antibiotics were associated with decreased rates of chorioamnionitis, delivery within 48 hours, and delivery within 7 days. Neonatal shortterm outcomes were also improved [70]. A similar benefit was noted with the utilization of antibiotics for asymptomatic bacteriuria in a 14 study meta-analysis conducted by Smail et al. While there was no clear reduction in preterm delivery, asymptomatic bacteriuria, pyelonephritis, and low birthweight were reduced [71]. As a result of this evidence supporting antibiotic treatment for these two conditionsas least for short-term outcomes - antibiotic prophylaxis and treatment is now routinely employed.

In addition, positive fetal fibronectin (FFN) is strongly associated with both infection and PTB. FFN leaks into the genital tract with disruption of the maternal-fetal interface through mechanisms such as infection, inflammation, and hemorrhage. Therefore randomized trials have evaluated the potential benefit of antibiotic therapy among those with a positive fetal fibronectin test. Andrews et al. randomized women to metronidazole or placebo. No differences were noted in SPTB <37 weeks (OR 1.17, 95\% CI: 0.8-1.70) or at earlier gestational age cutoffs [58].

In another trial by Shennan et al., asymptomatic women with positive fetal fibronectin were randomized to a oneweek course of metronidazole or placebo. Previous studies regarding metronidazole showed mixed results with regard to benefit in terms of preterm labor especially in patients with bacterial vaginosis. However, Shennan chose to study the effect of this antimicrobial in patients between 24-27 weeks of gestational age and with positive vaginal FFN. Patients randomized to metronidazole had an increased risk of preterm delivery less than 30 weeks (21\% versus $11 \%$, odds ratio $1.9,95 \% \mathrm{CI}: 0.72-5.09, P=0.18)$ as well as prior to 37 weeks (62\% versus $39 \%$, odds ratio $1.6,95 \%$ CI: $1.05-2.4$, $P=0.02)$. The trial was prematurely stopped. Thus, similar to the findings for $\mathrm{BV}$, treatment may increase the risk of preterm delivery [59].

\section{Conclusions}

There is no doubt that preterm birth continues to be an obstetrical and neonatal priority in the 21st century-with infection as a major cause. Trials evaluating antibiotic treatment to prevent PTB have yielded mixed results regarding benefit. No identifiable patterns regarding timing of antibiotic administration, gestational age at administration, antibiotic of choice, repeat dosages of antibiotics, the presence of documented underlying infection, or positive fetal fibronectin have emerged to suggest a role for these prophylactic antibiotics at this time. Moreover, a number of studies have suggested harm, and antibiotics should be avoided 
for prophylaxis for asymptomatic bacterial vaginosis and trichomoniasis. As such, routine antibiotic prophylaxis is not recommended for prevention of PTB. Based on the review, there may be promise of further studying the timing of antibiotic administration-even before conception-as well as more directed antimicrobial therapy. Studies targeted at timing of antibiotic therapy could be undertaken by treating patients not only during early pregnancy, but between pregnancies or shortly in the postpartum period with specific evaluation of the microbial = human flora interacrtion. Further studies aimed at elucidating the exact mechanism of decidual activation and the most-implicated microbes may be helpful to delineate specific antimicrobial agents and their optimal mode of delivery (oral versus vaginal versus parenteral). Furthermore, molecular receptor analysis, genetics, and proteomics may be the other steps needed to elucidate the black box that occurs between infection and preterm delivery.

\section{Conflict of Interests}

No financial conflict of interests are noted by the authors.

\section{References}

[1] E. M. Lewit, L. S. Baker, H. Corman, and P. H. Shiono, "The direct cost of low birth weight," The Future of Children, vol. 5, no. 1, pp. 35-56, 1995.

[2] M. M. Slattery and J. J. Morrison, "Preterm delivery," Lancet, vol. 360, no. 9344, pp. 1489-1497, 2002.

[3] B. E. Hamilton, J. A. Martin, and S. J. Ventura, Births: Preliminary Data for 2005, Health E-Stats, Hyattsville, Md, USA, 2006.

[4] J. A. Martin, B. E. Hamilton, S. J. Ventura, F. Menacker, M. M. Park, and P. D. Sutton, "Births: final data for 2001," National Vital Statistics Reports, vol. 51, no. 2, pp. 1-104, 2002.

[5] J. A. Martin, K. D. Kochanek, D. M. Strobino, B. Guyer, and M. F. MacDorman, "Annual summary of vital statistics-2003," Pediatrics, vol. 115, no. 3, pp. 619-634, 2005.

[6] J. A. Martin, B. E. Hamilton, P. D. Sutton et al., "Births: final data for 2007," National Vital Statistics Reports, vol. 58, no. 24, pp. 1-88, 2010.

[7] M. C. McCormick, "The contribution of low birth weight to infant mortality and childhood morbidity," New England Journal of Medicine, vol. 312, no. 2, pp. 82-90, 1985.

[8] R. L. Goldenberg, J. F. Culhane, J. D. Iams, and R. Romero, "Epidemiology and causes of preterm birth," The Lancet, vol. 371, no. 9606, pp. 75-84, 2008.

[9] J. M. Tucker, R. L. Goldenberg, R. O. Davis, R. L. Copper, C. L. Winkler, and J. C. Hauth, "Etiologies of preterm birth in an indigent population: is prevention a logical expectation?" Obstetrics and Gynecology, vol. 77, no. 3, pp. 343-347, 1991.

[10] J. G. Smulian, C. V. Ananth, W. L. Kinzler, E. Kontopoulos, and A. M. Vintzileos, "Twin deliveries in the United States over three decades: an age-period-cohort analysis," Obstetrics and Gynecology, vol. 104, no. 2, pp. 278-285, 2004.

[11] P. J. Steer, "The epidemiology of preterm labour-why have advances not equated to reduced incidence?" BJOG, vol. 113, supplement 3, pp. 1-3, 2006.
[12] R. L. Goldenberg, J. C. Hauth, and W. W. Andrews, "Intrauterine infection and preterm delivery," New England Journal of Medicine, vol. 342, no. 20, pp. 1500-1507, 2000.

[13] R. L. Goldenberg, W. W. Andrews, O. Faye-Petersen, S. Cliver, A. R. Goepfert, and J. C. Hauth, "The Alabama Preterm Birth Project: placental histology in recurrent spontaneous and indicated preterm birth," American Journal of Obstetrics and Gynecology, vol. 195, no. 3, pp. 792-796, 2006.

[14] R. Romero and M. Mazor, "Infection and preterm labor," Clinical Obstetrics and Gynecology, vol. 31, no. 3, pp. 553-584, 1988.

[15] W. W. Andrews, J. C. Hauth, R. L. Goldenberg, R. Gomez, R. Romero, and G. H. Cassell, "Amniotic fluid interleukin-6: correlation with upper genital tract microbial colonization and gestational age in women delivered after spontaneous labor versus indicated delivery," American Journal of Obstetrics and Gynecology, vol. 173, no. 2, pp. 606-612, 1995.

[16] R. Gomez, R. Romero, S. S. Edwin, and D. David, "Pathogenesis of preterm labor and preterm premature rupture of membranes associated with intraamniotic infection," Infectious Disease Clinics of North America, vol. 11, no. 1, pp. 135-176, 1997.

[17] G. Cassell, W. Andrews, J. Hauth et al., "Isolation of microorganisms from the chorioamnion is twice that from amniotic fluid at cesarean delivery in women with intact membranes," American Journal of Obstetrics \& Gynecology, pp. 168-424, 1993.

[18] K. J. Arntzen, A. M. Kjøllesdal, J. Halgunset, L. Vatten, and R. Austgulen, "TNF, IL-1, IL-6, IL-8 and soluble TNF receptors in relation to chorioamnionitis and premature labor," Journal of Perinatal Medicine, vol. 26, no. 1, pp. 17-26, 1998.

[19] R. Romero, M. Mazor, W. Sepulveda, C. Avila, D. Copeland, and J. Williams, "Tumor necrosis factor in preterm and term labor," American Journal of Obstetrics and Gynecology, vol. 166, no. 5, pp. 1576-1587, 1992.

[20] Y. Tanaka, H. Narahara, N. Takai, J. Yoshimatsu, T. Anal, and I. Miyakawa, "Interleukin- $1 \beta$ and interleukin- 8 in cervicovaginal fluid during pregnancy," American Journal of Obstetrics and Gynecology, vol. 179, no. 3, pp. 644-649, 1998.

[21] M. Winkler, D. C. Fischer, M. Hlubek et al., "Interleukin-1ß and interluekin- 8 concentrations in the lower uterine segment during parturition at term," Obstetrics \& Gynecology, vol. 91, pp. 945-949, 1998.

[22] R. Romero, R. Gomez, F. Ghezzi et al., "A fetal systemic inflammatory response is followed by the spontaneous onset of preterm parturition," American Journal of Obstetrics and Gynecology, vol. 179, no. 1, pp. 186-193, 1998.

[23] W. W. Andrews, J. C. Hauth, R. L. Goldenberg, R. Gomez, R. Romero, and G. H. Cassell, "Amniotic fluid interleukin-6: correlation with upper genital tract microbial colonization and gestational age in women delivered after spontaneous labor versus indicated delivery," American Journal of Obstetrics and Gynecology, vol. 173, no. 2, pp. 606-612, 1995.

[24] W. W. Andrews, J. C. Hauth, and R. L. Goldenberg, "Infection and preterm birth," American Journal of Perinatology, vol. 17, no. 7, pp. 357-365, 2000.

[25] S. L. Hillier, J. Martius, M. Krohn, N. Kiviat, K. K. Holmes, and D. A. Eschenbach, "A case-control study of chorioamnionic infection and histologic chorioamnionitis in prematurity," New England Journal of Medicine, vol. 319, no. 15, pp. 972978, 1988.

[26] W. W. Andrews, R. L. Goldenberg, and J. C. Hauth, "Preterm labor: emerging role of genital tract infections," Infectious Agents and Disease, vol. 4, no. 4, pp. 196-211, 1995. 
[27] R. S. Gibbs, R. Romero, S. L. Hillier, D. A. Eschenbach, and R. L. Sweet, "A review of premature birth and subclinical infection," American Journal of Obstetrics and Gynecology, vol. 166, no. 5, pp. 1515-1528, 1992.

[28] M. A. Krohn, S. L. Hillier, R. P. Nugent et al., "The genital flora of women with intraamniotic infection," Journal of Infectious Diseases, vol. 171, no. 6, pp. 1475-1480, 1995.

[29] R. L. Goldenberg, W. W. Andrews, A. R. Goepfert et al., "The Alabama Preterm Birth Study: umbilical cord blood Ureaplasma urealyticum and Mycoplasma hominis cultures in very preterm newborn infants," American Journal of Obstetrics and Gynecology, vol. 198, no. 1, pp. 43e1-43e5, 2008.

[30] I. Hendler, W. W. Andrews, C. J. Carey et al., "The relationship between resolution of asymptomatic bacterial vaginosis and spontaneous preterm birth in fetal fibronectin-positive women," American Journal of Obstetrics and Gynecology, vol. 197, no. 5, pp. 488.e1-488.e5, 2007.

[31] R. L. Goldenberg, J. D. Iams, B. M. Mercer et al., "The preterm prediction study: the value of new vs standard risk factors in predicting early and all spontaneous preterm births," American Journal of Public Health, vol. 88, no. 2, pp. 233-238, 1998.

[32] C. A. Flynn, A. L. Helwig, and L. N. Meurer, "Bacterial vaginosis in pregnancy and the risk of prematurity: a metaanalysis," Journal of Family Practice, vol. 48, no. 11, pp. 885892, 1999.

[33] H. Leitich, B. Bodner-Adler, M. Brunbauer, A. Kaider, C. Egarter, and P. Husslein, "Bacterial vaginosis as a risk factor for preterm delivery: a meta-analysis," American Journal of $\mathrm{Ob}$ stetrics and Gynecology, vol. 189, no. 1, pp. 139-147, 2003.

[34] M. F. Cotch, J. G. Pastorek, R. P. Nugent et al., “Trichomonas vaginalis associated with low birth weight and preterm delivery," Sexually Transmitted Diseases, vol. 24, no. 6, pp. 353360, 1997.

[35] R. L. Sweet, D. L. Landers, C. Walker, and J. Schachter, "Chlamydia trachomatic infection and pregnancy outcome," American Journal of Obstetrics \& Gynecology, vol. 156, pp. 824 833, 1987.

[36] G. G. Donders, J. Desmyter, D. H. de Wet, and F. A. van Assche, "The association of gonorrhea and syphilis with premature birth and low birth weight," Genitourinary Medicine, vol. 69, pp. 98-101, 1993.

[37] R. L. Goldenberg, J. F. Culhane, and D. C. Johnson, "Maternal infection and adverse fetal and neonatal outcomes," Clinics in Perinatology, vol. 32, no. 3, pp. 523-559, 2005.

[38] R. Romero, E. Oyarzun, M. Mazor, M. Sirtori, J. C. Hobbins, and M. Bracken, "Meta-analysis of the relationship between asymptomatic bacteriuria and preterm delivery/low birth weight," Obstetrics and Gynecology, vol. 73, no. 4, pp. 576-582, 1989.

[39] J. M. B. Hardy, E. N. Azarowicz, A. Mannini et al., "The effect of Asian influenza on the outcome of pregnancy. Baltimore 1957-1958," American Journal of Public Health, vol. 51, pp. 1182-1188, 1961.

[40] P. Horn, "Poliomyelitis in pregnancy; a twenty-year report from Los Angeles County, California," Obstetrics and gynecology, vol. 6, no. 2, pp. 121-137, 1955.

[41] E. R. Newton, L. Shields, L. E. Ridgway, M. D. Berkus, and B. D. Elliott, "Combination antibiotics and indomethacin in idiopathic preterm labor: a randomized double-blind clinical trial," American Journal of Obstetrics and Gynecology, vol. 165, no. 6, pp. 1753-1759, 1991.
[42] S. M. Cox, V. R. Bohman, L. Sherman, and K. J. Leveno, "Randomized investigation of antimicrobials for the prevention of preterm birth," American Journal of Obstetrics and Gynecology, vol. 174, no. 1, pp. 206-210, 1996.

[43] M. Gordon, P. Samuels, P. Shubert et al., "A randomized, prospective study of adjunctive ceftizosime in preterm labor," American Journal of Obstetrics and Gynecology, vol. 172, no. 5, pp. 1546-1552, 1995.

[44] J. A. McGregor, J. I. French, and K. Seo, "Adjunctive clindamycin therapy for preterm labor: results of a double-blind, placebo-controlled trial," American Journal of Obstetrics and Gynecology, vol. 164, no. 1, p. 259, 1991.

[45] R. F. Lamont, S. L. B. Duncan, D. Mandal, and P. Bassett, "Intravaginal clindamycin to reduce preterm birth in women with abnormal genital tract flora," Obstetrics and Gynecology, vol. 101, no. 3, pp. 516-522, 2003.

[46] M. Kekki, T. Kurki, J. Pelkonen, M. Kurkinen-Räty, B. Cacciatore, and J. Paavonen, "Vaginal clindamycin in preventing preterm birth and peripartal infections in asymptomatic women with bacterial vaginosis: a randomized, controlled trial," Obstetrics and Gynecology, vol. 97, no. 5, pp. 643-648, 2001.

[47] N. R. van den Broek, S. A. White, M. Goodall et al., "The APPLe study: a randomized, community-based, placebo-controlled trial of azithromycin for the prevention of preterm birth, with meta-analysis," PLoS Medicine, vol. 6, no. 12, article e1000191, 2009.

[48] K. Norman, R. C. Pattinson, J. de Souza, P. de Jong, G. Moller, and G. Kirsten, "Ampicillin and metronidazole treatment in preterm labour: a multicentre, randomised controlled trial," British Journal of Obstetrics and Gynaecology, vol. 101, no. 5, pp. 404-408, 1994.

[49] J. Svare, J. Langhoff-Roos, L. F. Andersen et al., "Ampicillinmetronidazole treatment in idiopathic preterm labour: a randomised controlled multicentre trial," British Journal of Obstetrics and Gynaecology, vol. 104, no. 8, pp. 892-897, 1997.

[50] E. R. Newton, M. J. Dinsmoor, and R. S. Gibbs, "A randomized, blinded, placebo-controlled trial of antibiotics in idiopathic preterm labor," Obstetrics and Gynecology, vol. 74, no. 4, pp. 562-566, 1989.

[51] R. Romero, B. Sibai, S. Caritis et al., "Antibiotic treatment of preterm labor with intact membranes: a multicenter, randomized, double-blinded, placebo-controlled trial," American Journal of Obstetrics and Gynecology, vol. 169, no. 4, pp. 764$774,1993$.

[52] S. L. Kenyon, D. J. Taylor, and W. Tarnow-Mordi, "Broadspectrum antibiotics for spontaneous preterm labour: the ORACLE II randomised trial," Lancet, vol. 357, no. 9261, pp. 991-996, 2001.

[53] J. C. Hauth, R. L. Goldenberg, W. W. Andrews, M. B. Dubard, and R. L. Copper, "Reduced incidence of preterm delivery with metronidazole and erythromycin in women with bacterial vaginosis," New England Journal of Medicine, vol. 333, no. 26, pp. 1732-1736, 1995.

[54] H. M. McDonald, J. A. O'Loughlin, R. Vigneswaran et al., "Impact of metronidazole therapy on preterm birth in women with bacterial vaginosis flora (Gardnerella vaginalis): a randomised, placebo controlled trial," British Journal of Obstetrics and Gynaecology, vol. 104, no. 12, pp. 1391-1397, 1997.

[55] M. A. Klebanoff, J. C. Carey, J. C. Hauth et al., "Failure of metronidazole to prevent preterm delivery among pregnant women with asymptomatic Trichomonas vaginalis infection," New England Journal of Medicine, vol. 345, no. 7, pp. 487-493, 2001. 
[56] A. Ugwumadu, I. Manyonda, F. Reid, and P. Hay, "Effect of early oral clindamycin on late miscarriage and preterm delivery in asymptomatic women with abnormal vaginal flora and bacterial vaginosis: a randomised controlled trial," Lancet, vol. 361, no. 9362, pp. 983-988, 2003.

[57] G. G. Kigozi, H. Brahmbhatt, F. Wabwire-Mangen et al., "Treatment of Trichomonas in pregnancy and adverse outcomes of pregnancy: a subanalysis of a randomized trial in Rakai, Uganda," American Journal of Obstetrics and Gynecology, vol. 189, no. 5, pp. 1398-1400, 2003.

[58] W. W. Andrews, B. M. Sibai, E. A. Thom et al., "Randomized clinical trial of metronidazole plus erythromycin to prevent spontaneous preterm delivery in fetal fibronectin-positive women," Obstetrics and Gynecology, vol. 101, no. 5, pp. 847855, 2003.

[59] A. Shennan, S. Crawshaw, A. Briley et al., "A randomised controlled trial of metronidazole for the prevention of preterm birth in women positive for cervicovaginal fetal fibronectin: the PREMET Study," BJOG, vol. 113, no. 1, pp. 65-74, 2006.

[60] J. A. Macgregor and J. I. French, "Evidence-based prevention of preterm birth and rupture of membranes: infection and inflammation," Society of Obstetricians and Gynaecologists of Canada, vol. 19, pp. 835-852, 1997.

[61] G. M. Vermeulen and H. W. Bruinse, "Prophylactic administration of clindamycin $2 \%$ vaginal cream to reduce the incidence of spontaneous preterm birth in women with an increased recurrence risk: a randomized placebo-controlled double-blind trial," British Journal of Obstetrics and Gynaecology, vol. 106, no. 7, pp. 652-657, 1999.

[62] M. Kurkinen-Raty, S. Vuopala, M. Koskela et al., "A randomised controlled trial of vaginal clindamycin for early pregnancy bacterial vaginosis," British Journal of Obstetrics and Gynaecology, vol. 107, no. 11, pp. 1427-1432, 2000.

[63] D. H. Watts, M. A. Krohn, S. L. Hillier, and D. A. Eschenbach, "Randomized trial of antibiotics in addition to tocolytic therapy to treat preterm labor," Infectious Diseases in Obstetrics and Gynecology, vol. 1, no. 5, pp. 220-227, 1994.

[64] E. Oyarzún, R. Gómez, A. Rioseco et al., "Antibiotic treatment in preterm labor and intact membranes: a randomized, double-blinded, placebo-controlled trial," Journal of Maternal-Fetal and Neonatal Medicine, vol. 7, no. 3, pp. 105110, 1998.

[65] J. F. King and V. Flenady, "Prophylactic antibiotics for inhibiting preterm labour with intact membranes," Cochrane Database of Systematic Reviews, no. 4, Article ID CD000246, 2002.

[66] R. Simcox, W. T. Sin, P. T. Seed, A. Briley, and A. H. Shennan, "Prophylactic antibiotics for the prevention of preterm birth in women at risk: a meta-analysis," Australian and New Zealand Journal of Obstetrics and Gynaecology, vol. 47, no. 5, pp. 368-377, 2007.

[67] J. C. Carey, M. A. Klebanoff, J. C. Hauth et al., "Metronidazole to prevent preterm delivery in pregnant women with asymptomatic bacterial vaginosis. National Institute of Child Health and Human Development Network of Maternal-Fetal Medicine Units," New England Journal of Medicine, vol. 342, no. 8, pp. 534-540, 2000.

[68] H. M. McDonald, P. Brocklehurst, and A. Gordon, "Antibiotics for treating bacterial vaginosis in pregnancy," Cochrane Database of Systematic Reviews, no. 1, Article ID CD000262, 2007.

[69] W. W. Andrews, R. L. Goldenberg, J. C. Hauth, S. P. Cliver, R. Copper, and M. Conner, "Interconceptional antibiotics to prevent spontaneous preterm birth: a randomized clinical trial," American Journal of Obstetrics and Gynecology, vol. 194, no. 3, pp. 617-623, 2006.

[70] S. Kenyon, M. Boulvain, and J. P. Neilson, "Antibiotics for preterm rupture of membranes," Cochrane Database of Systematic Reviews, vol. 8, Article ID CD001058, 2010.

[71] F. Smaill and J. C. Vazquez, "Antibiotics for asymptomatic bacteriuria in pregnancy," Cochrane Database of Systematic Reviews, no. 2, Article ID CD000490, 2007. 


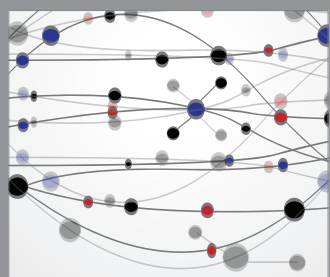

The Scientific World Journal
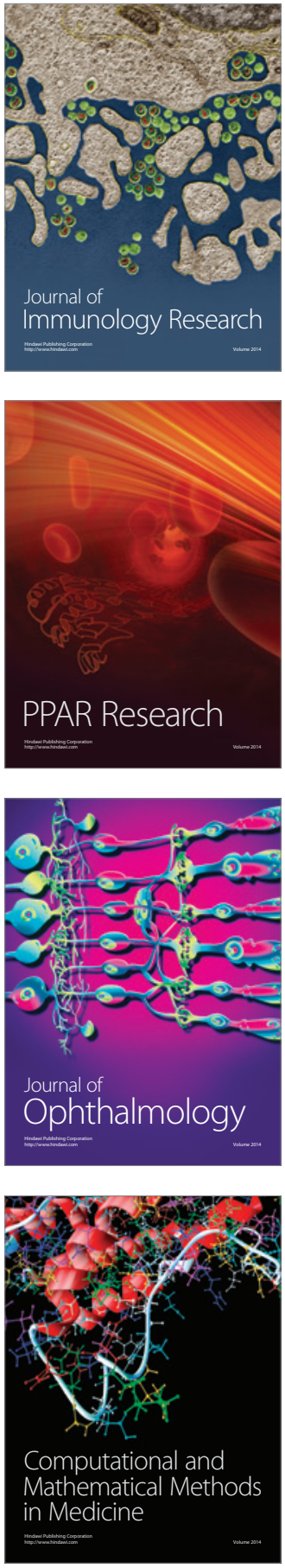

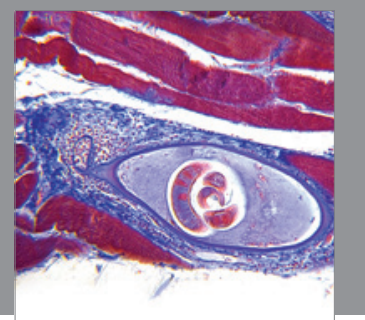

Gastroenterology

Research and Practice
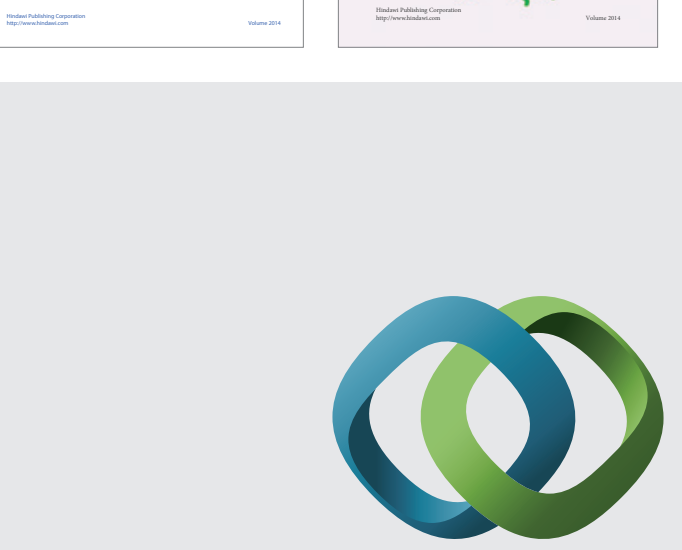

\section{Hindawi}

Submit your manuscripts at

http://www.hindawi.com
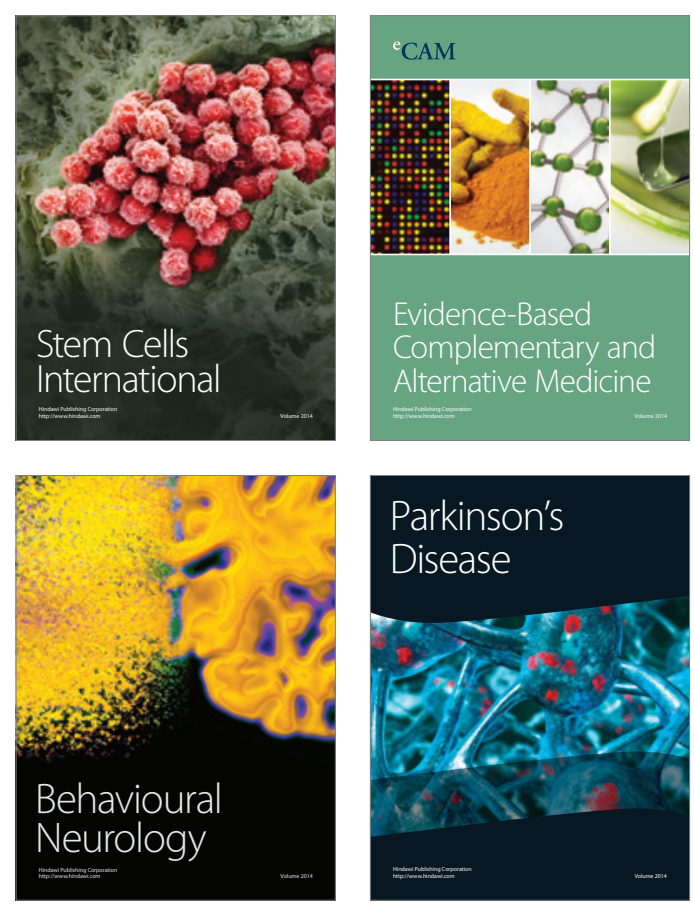

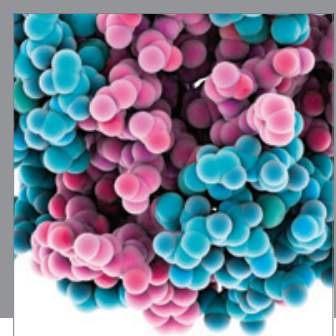

Journal of
Diabetes Research

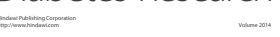

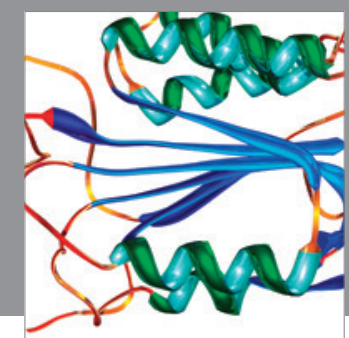

Disease Markers
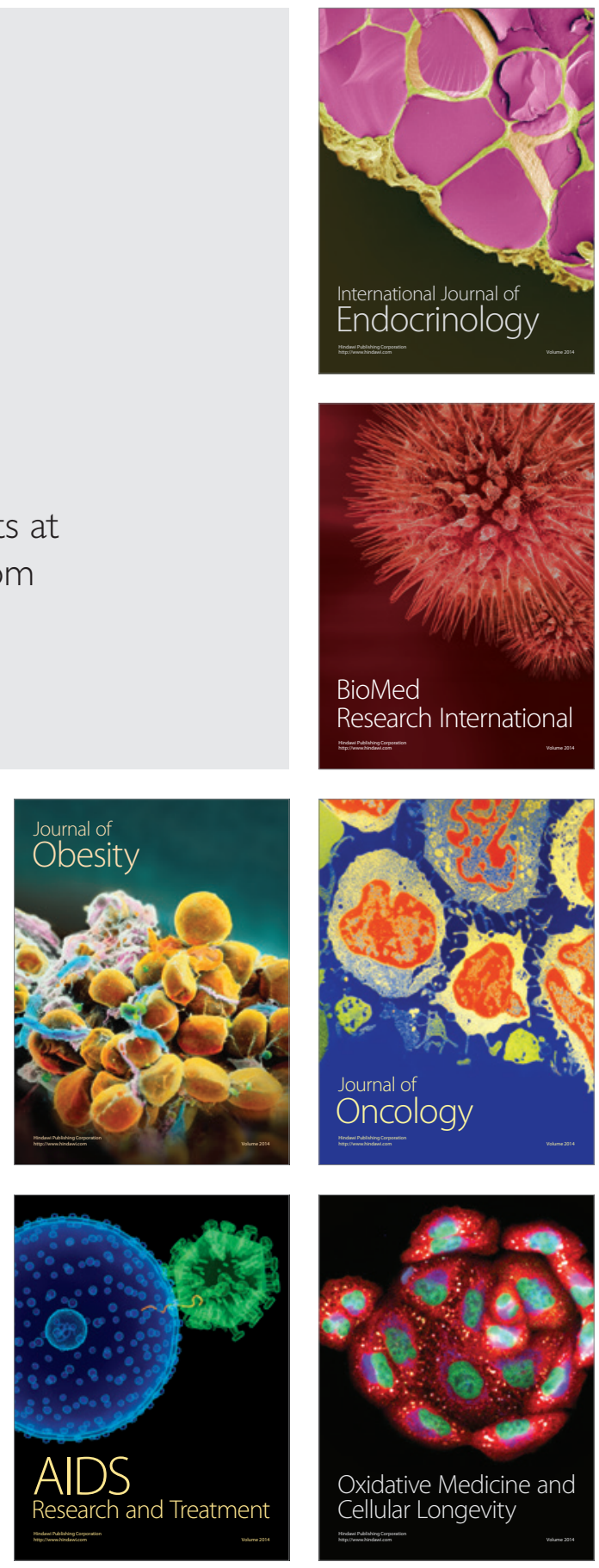